\title{
Distortion-oriented Workspace Awareness in DOME
}

\section{Philip Weir \& Andy Cockburn}

Department of Computer Science, University of Canterbury, Christchurch, New Zealand.

Tel: $+6433642987 \times 7768$

Fax: +6433642569

EMail:weirp@acm.org,andy@cosc.canterbury.ac.nz

URL: http://www.cosc.canterbury.ac.nz/andy/

Distortion-oriented visualization techniques such as magnification-lenses, zooming functions and fish-eye views are useful in a wide range of singleuser computing systems. They assist visualization of large information spaces by easing the transition between high-levels of detail in a local area of interest and the global context of the information space.

In real-time groupware environments, distortion-oriented visualizations offer additional benefits. By providing one distorted region for each user of a groupware workspace, users can maintain an awareness of the location and activities of their colleagues while simultaneously having a focused area of detail for their own work.

We describe the design and evaluation of DOME, a fully-functional distortion-oriented multi-user editor. Unexpected usability problems and potential solutions are discussed.

Keywords: groupware, collaborative workspace awareness, distortionoriented visualization. 


\section{Introduction}

One of the major problems confronting groupware designers is how to provide collaborative awareness between simultaneous users of a groupware information space. The simplest solution is to enforce strict-WYSIWIS (what you see is what I see) on the displays of all users. Strict-WYSIWIS has beneficial properties: it is relatively easy to implement and it is conceptually easy to use in that all users have precisely the same view. Unfortunately, it is a highly constraining style of collaboration in which the users are forced to work as a tightly coupled unit. This constraint fails to allow natural and dynamic styles of collaboration in which colleagues fluidly move between periods of close collaboration and periods of more autonomous work.

Experiments with relaxing the strict-WYSIWIS paradigm in order to increase the extent of user support have resulted in difficulties with users and designers misunderstanding the implications of the relaxations (Stefik et al., 1987). These difficulties, which manifest themselves in collaboration breakdowns such as mistaken deictic reference*, are largely due to the reduced mutual workspace awareness of colleagues' location and activities.

The problem, then, is to simultaneously provide users with a representation of their local area of work while simultaneously providing the 'right amount' of awareness of colleagues' location and activities. Current approaches to facilitating workspace awareness in synchronous groupware environments focus on 'awareness widgets' such as radar views (Gutwin et al., 1996) which provide renderings of the entire workspace within a miniature overview window; each user's location of activity is superimposed on the overview. The problems with miniaturization techniques are that they have limited scalability (a miniature rendering of an extremely large data-space will yield too little detail to be useful) and they provide a static level of detail, consequently limiting each user's ability to customize their awareness of colleagues.

In this paper we propose that distortion-oriented visualization techniques are a natural candidate for providing collaborative workspace awareness. In single-user systems distortion-oriented visualizations such as magnification-lenses, zooming functions and fish-eye views ease the transition between high-levels of detail in a local area of interest and the global context of the information space. In groupware environments they can be used to provide tailorable levels of awareness of coworkers while maintaining a focus on the local region of personal work. Our previous work (Greenberg et al., 1996a; Greenberg et al., 1996b; Greenberg et al., 1996c) has demonstrated several styles of distortion-oriented workspace awareness using point systems, but it has fallen short of evaluation. In this paper we describe a fully functional and robust distortion-oriented multi-user editor called DOME, and we discuss the results of its evaluation.

Section 2 describes DOME and its workspace awareness capabilities. Section 3 details the evaluation of DOME, and Section 4 discusses the findings of the evaluation and identifies directions for further work. Related work is reviewed in Section 5, and Section 6 concludes the paper.

*Deictic reference is a combination of reference by gesture and verbal reference such as 'this one' (Stefik et al., 1987). 


\section{DOME: A Distortion-Oriented Multi-user Editor}

We built DOME to experiment with the effectiveness of distortion-oriented techniques for supporting workspace awareness in synchronous groupware environments. We strongly believe that in order to test the effectiveness of distortion-oriented workspace awareness it is necessary to provide a full-functionality and robust environment that offers a polished and professional interface. Point systems with minimalist interfaces such as those constructed as part of our earlier research (see Section 1) are inadequate for realistic usability evaluation ${ }^{\dagger}$.

DOME's main window is shown in Figure 1. As a single-user system it is a functionally rich text-editing environment that supports extensive facilities for annotation (described in Section 5) as well as the normal text-editing facilities such as undo, cut, copy and paste. $\mathrm{AT}_{\mathrm{E}} \mathrm{X}$ documents and $\mathrm{TCl} / \mathrm{Tk}$ programs are automatically parsed and their structure can be displayed in a graphical tree that allows shortcuts to portions of the document or program.

In the sections below we describe several of DOME's groupware support facilities: the workspace awareness provided by its fisheye views, its support for tightly coupled collaboration, and its support for loose collaboration through shared and private annotations.

\subsection{Fisheye Views for Tailorable Workspace Awareness}

Fisheye views were first proposed by Furnas (1986) to provide a 'focus plus context' view of information spaces. Visualizations of the data-space are distorted according to the users 'degree of interest' in regions of the information space. Related work on the fisheye view concept is discussed in Section 5.

In DoME each user has a fisheye view on their own region of work. Text within the fisheye is magnified or demagnified according to the configuration of their lens. Each user also has one fisheye view for each of the simultaneous users of the system. The design intention is that each user can control the amount of screen real-estate dedicated to their own area of interest, while simultaneously maintaining a tailorable level of awareness on the location and activities of their colleagues.

Each tailorable fisheye lens controls the degree and range of magnification. The degree of magnification or demagnification (the size of the text font) at any point within a lens is dependent upon the vertical distance that the text lies from the centre of a fisheye lens. The centre of each lens follows the location of the user's insertion cursor while typing. Users can also move their lens independently of the insertion cursor in the same way that most word processors allow users to scroll to other regions in the document without disrupting the location of the insertion point. Figure 2 provides an extreme example of the stepped magnification provided by one of DOME's lenses: the three central lines are very large and bold; there are two outer levels of magnification, each of 4 lines and each with slightly lower levels of magnification. It is important to note that each user's customization of their lenses (both their own lens, and their lenses onto their colleagues' workspaces) does not affect the other users' displays.

$\dagger$ This paper was written using DOME and the LATEX text formatting and typesetting system. 


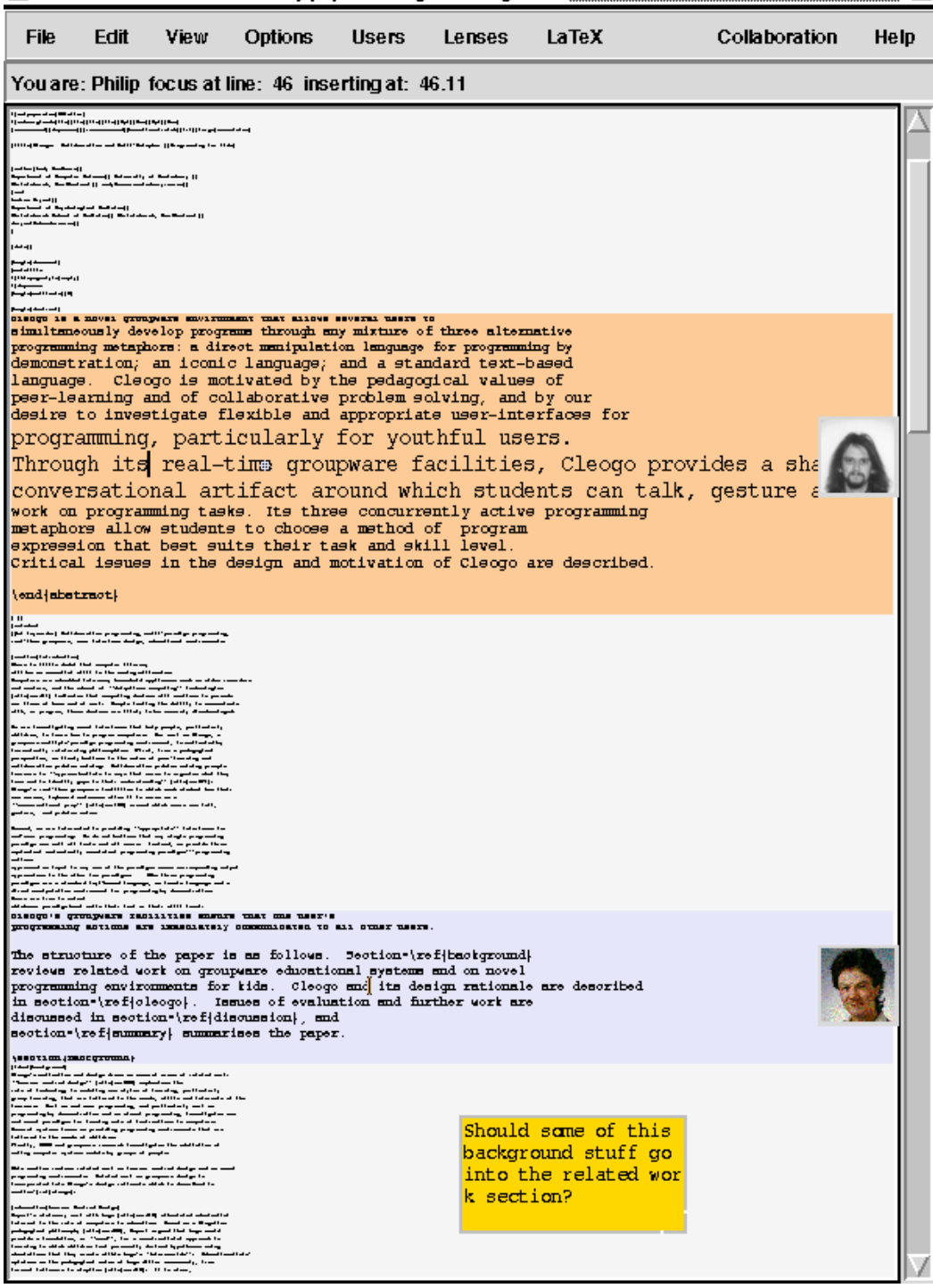

Figure 1: DoME's main window. 


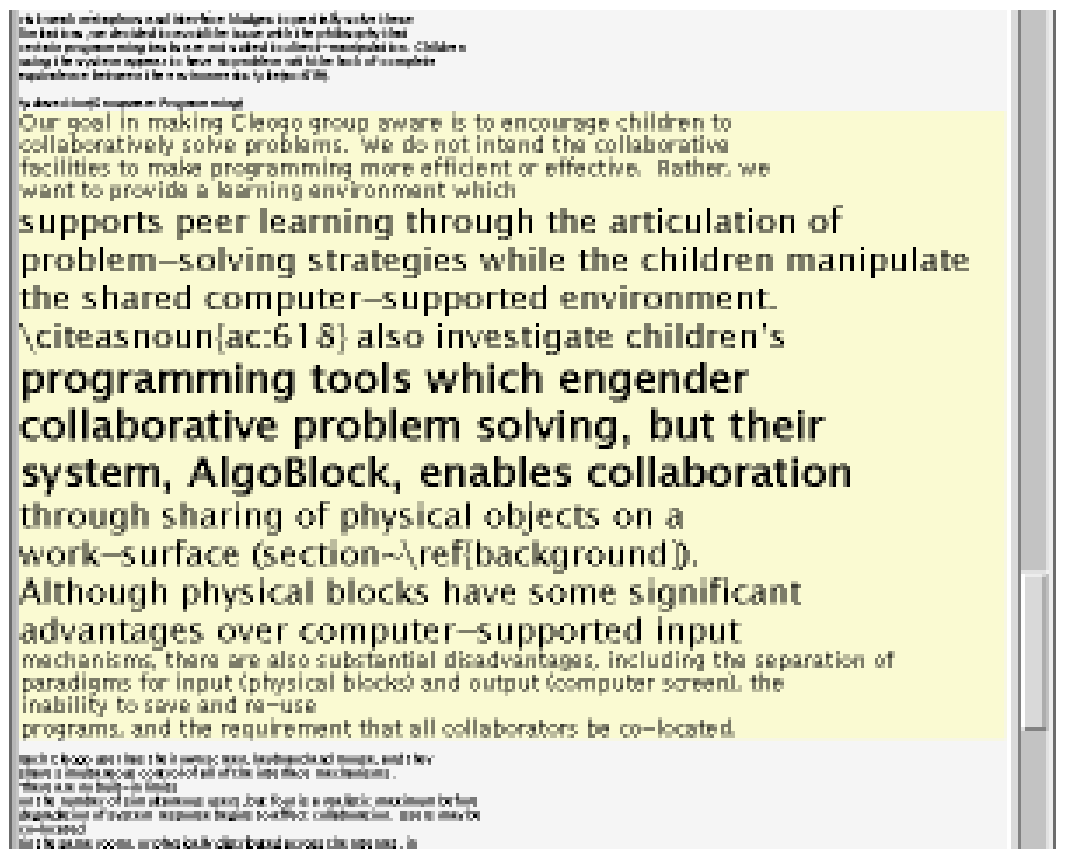

Figure 2: An extreme example of a lens's stepped magnification.

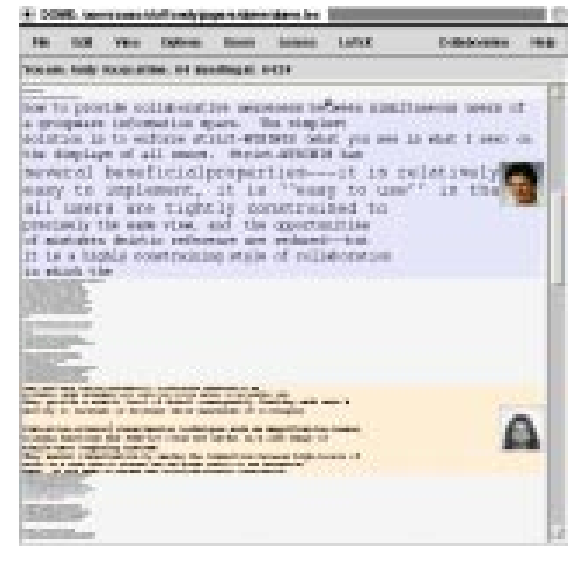

(a) Andy's view.

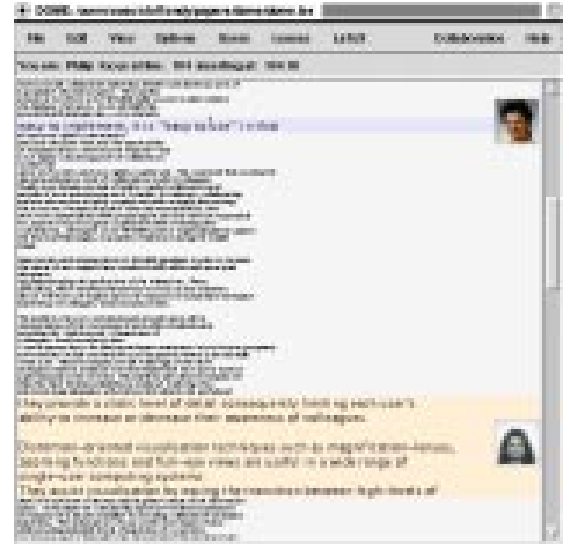

(b) Philip's view.

Figure 3: Two simultaneous views of the workspace. 


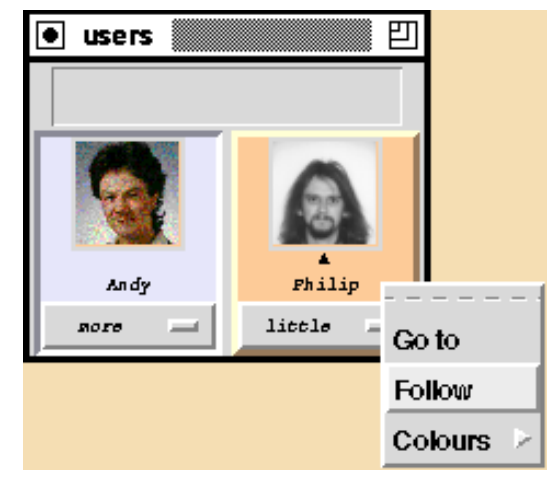

Figure 4: Initiating tightly coupled collaboration in the 'Users' window.

Figures 3(a) \& 3(b) show two users' simultaneous views of the document workspace using DOME. In the left-hand figure, Andy has configured his lenses so that he has a large legible region for his own text-area; his view onto Philip's work area is configured to provide a roughly paragraph-size legible viewport; and he has configured the background text to a sufficiently small size to give an extensive overall view of the document. In the right-hand figure, Philip has simultaneously configured his lens to provide a small region for his local text area, just one line for his view on Andy's work, and a just-legible font for the background text.

User configurable colours are used as an additional cue to the text region contained within each fisheye lens. Colours are used consistently across all users displays - if, for example, purple is used to denote Mary's region on one person's display, then Mary's region is purple on all displays. Consistent colour coding is intended to reduce the opportunity for mistaken deictic reference in expressions such as "the red bit of text at the top".

\subsection{Tailoring Levels of Awareness}

One of the major design intentions with DOME is to ease each user's transition between varying levels of awareness of colleagues.

There are several ways to configure awareness of specific users, but the most common way is through the 'users' window (Figure 4). This window shows an image of each user, the colour of their text region and their awareness level. There is also a menu under each user's image which allows the fisheye onto that user's region of activity to be set to one of six discrete awareness levels (from 'None' to 'Very High'). The precise configuration of the lens under each level can be tailored using 'lens handles' which allow the user to change a variety of lens properties, including the number of stepped magnification levels it provides, the range (number of lines) within each level, and the amount of magnification within each level. The default awareness level for new arrivals into a shared editing session can be tailored.

The discrete awareness levels allow users to rapidly move between, for instance, high levels of awareness for tightly coupled collaborative activity and low levels of awareness (or none) for more independent work. 
The awareness features in DoME are highly configurable. Some of the configuration options are primarily intended to support our evaluation of different mechanisms for awareness, but others are intended to support user preferences, such as whether to display each user's image at their focal point.

\subsection{Tightly Coupled Interaction}

During tightly coupled collaborative work, 'awareness' of a colleague's activities may be insufficient. At such times users may need to revert to a strict-WYSIWIS mode of working in which all movements, actions and gestures are communicated between the users.

DOME provides four facilities to assist tightly coupled work. First, using the menu shown in Figure 4, a user can 'Goto' the location of any other user, causing their window to immediately display the location of their colleague. Second, a user may choose to 'Follow' another user, which slaves the user's screen location to that of the 'followed' user. Third, DomE provides 'semantic telepointers' (Greenberg et al., 1996d) which communicate to other users the region of text that a user points at with their mouse. The semantic telepointers assure that if one user points at a particular word, then his or her telepointer on the other users' displays will also point at that word. This is a significant improvement on geometry-based telepointers which simply point to the same window coordinates without accounting for differing window sizes, variations in word-wrap and non-aligned scroll locations. Finally, when a user selects a region of text, the selection is communicated to all other users by shading the text region.

\subsection{Annotation}

The workspace awareness facilities described above are primarily intended to support the users' dynamic awareness of the location and activities of colleagues who are concurrently working within the workspace. Issues of temporal awareness - the awareness of what information has changed in the workspace - are also important.

DOME provides powerful annotation facilities which can be used for a variety of purposes including temporal awareness of document modifications and metacomments about the underlying text. Annotations in DOME are based on a 'stickynote' metaphor that closely maps to common paper-based editing practices. DoME's annotations provide extensions beyond the real-world metaphor to assist a variety of collaborative and personal editing strategies.

Annotations are created as either 'private' or 'shared'. Shared annotations are simultaneously editable by all users. Private annotations are shown only on the creator's window, but they can be shared between sub-groups of users (or all users) by sending them to others (see Figure 5).

Annotations can either be associated with a specific piece of text ('pinned to the text') or they can be unpinned and 'float' above the text with a constant geometry in the window regardless of the scrolled location. Pinned annotations are useful for comments about specific pieces of text (for instance, changes that have been made or should be made), and unpinned annotations are useful as general communication portholes between users. 


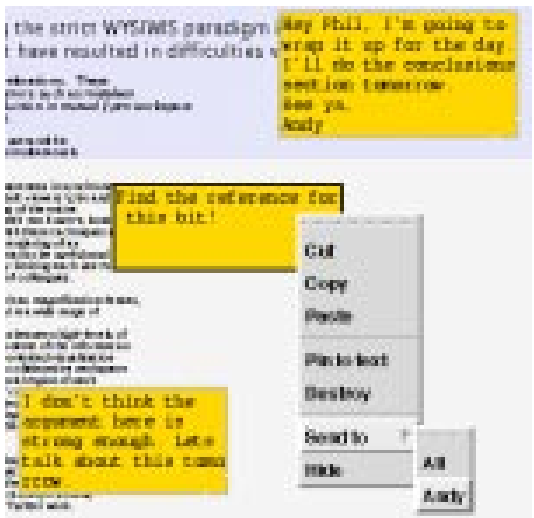

Figure 5: Three annotations: sending a private annotation to another user.

\section{Evaluation}

A primary design intention with DOME was to create a thoroughly polished editing environment that could be used to evaluate distortion-oriented workspace awareness. We strongly felt that the rudimentary interfaces of the demonstration systems used in our prior work (see Section 1) would obscure any evaluation.

The fourteen subjects in the evaluation were all postgraduate Computer Science students and Computer Science academics, and were randomly assigned to pairs. All subjects were familiar with the concepts of groupware systems, but none had previous experience with DOME. Each pair participated in a single one-hour videotaped session. During the evaluations the users were seated alongside one-another, approximately one and a half metres apart. We selected the side-by-side setting to illuminate failings in the workspace awareness: if users frequently looked at their partner's screen, it would indicate a failing in the awareness features.

In each session approximately twenty five minutes was spent introducing DOME's features and familiarizing the subjects with the distortion-oriented views. Although this is a relatively long time to dedicate to familiarization, we felt it was worthwhile to ensure that users were settled to the concept of the fisheye view prior to engaging in the evaluation tasks. Fifteen minutes were then spent on each of two tasks, and five minutes were allocated to a post task interview.

The first task involved re-ordering a jumbled structured document. It was introduced to the users as follows:

A production of "The Tragedy of Macbeth" by William Shakespeare is due to be performed tonight. The problem is that the script was emailed to the production company in parts, and assembled automatically by an email reassembler. The software malfunctioned and assembled the messages based on the order in which they arrived, rather than the sequence in which they were sent. 
Your task is to work together with your partner to reassemble the text so the play can go on. The file macbeth. tex contains the jumbled text.

In the second task the subjects were given a template document and were asked to complete the sections, and in particular to summarize these sections in a table at the end of the template. The task was introduced as follows:

Your second task is to fill-in the details of a document on the fourth year Computer Science papers offered in our department. The structure of the paper is provided in the file course. tex. Be sure to complete the summary table at the end of the document.

The two tasks were selected to generate differing requirements for workspace awareness. The first task was intended to require frequent transitions between collaborative work and independent work as the users moved from negotiating which scene to collect next (which we presumed would be a collaborative activity) to retrieving and repositioning the text (which we presumed would be an independent activity). The second task was selected to generate a need for workspace awareness in more tightly coupled collaborative activity. Although some of the sections in the template could be completed independently, we believed it would be necessary to collaborate in order to produce the summary table.

\subsection{Observations}

Overall the subjects' reactions to the distortion-oriented workspace awareness features were negative. The subjects reported, and we observed, some substantial limitations of the fisheye mechanisms which will have to be amended before subsequent evaluation. These difficulties are reported in the following sections.

Not all of our observations were negative, and there were many interesting and promising uses of the awareness features. During the second task, in which the subjects were writing about courses and lecturers, we noted several occurrences of subjects laughing. When asked, the subjects reported that they had seen what their partner had written about a particular course or lecturer. Although the information communicated through the awareness facilities was frivolous (in this case), it demonstrates that the subjects were aware of each others actions. Several of the pairs also made successful use of the shared annotations for mediating collaborative activity, such as keeping track of assigned tasks.

\subsection{1 'Jumping' Text caused by Fish-eyes}

The biggest problem, reported by all of the subjects, was caused by the small amount of unwanted text-motion that occurs when the fisheye lenses are moved. This problem arises as a result of two types of user action.

First, when a user moves their own insertion point their fisheye view moves to the new location. The text around the new location is immediately magnified according to the current configuration of their lens. This causes a small offset (both horizontal and vertical) between the coordinate clicked and the resultant location of the I-beam insertion point. The magnitudes of the horizontal and vertical offsets, which are formally expressed in Equations $1 \& 2$, show that the offsets are caused by 


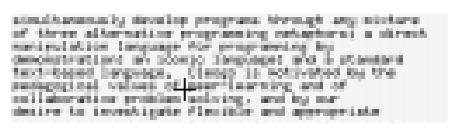

(a) Text before clicking. zanipulation larguage for prograrming by

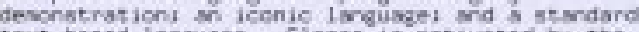

tent-based isnouage. Cieoso is notavated by the

pedidasical values of posr-leaming and of

cullabarative problen bolvirg. and by war

desire to inwestigote Rexible and speroperiste

uber-interfaceotif or programing. partioularly for youthrol uost... Through ite real-ting sroupuare

Figure 6: Unwanted text motion when repositioning the insertion point. The I-beam is displaced from the clicked coordinate.

$$
\delta_{x}=\sum_{c=0}^{\text {clickChar }} w_{R}(c)-w_{I}(c)
$$

where:

- $\delta_{x}$ is the horizontal offset between the clicked location and the resultant location of the I-beam.

- $c$ takes the value of each character from the start of the line to the clicked character.

- $w_{R}(x)$ gives the width of character $x$ at the resultant (magnified) font-size. Note that this value is the same for all characters in fixed-width fonts.

- $w_{I}(x)$ gives the width of character $x$ at the initial (background) font-size.

$$
\delta_{y}= \pm\left((h(1)-h(b)) * \frac{\text { lines }(1)}{2}+\sum_{l=2}^{\text {numLevels }}(h(l)-h(b)) * \text { lines }(l)\right)
$$

where:

- $\delta_{y}$ is the vertical offset between the clicked location and the resultant location of the I-beam. $\delta_{y}$ is negative (the I-beam appears below the clicked location) when the user clicks a text location above the current location of the fisheye. It is positive when the user clicks below the fisheye.

- $h(x)$ gives the font-height at magnification level $x$.

- $b$ is the background magnification level.

- $l$ takes one value for each of the stepped lens levels of magnification, from the 2nd stepped level to the outer-most level. Level 1 is the lenses centre of magnification.

- lines $(x)$ gives the number of lines contained within lens magnification level $x$.

Equations 1 \& 2: The horizontal and vertical offsets between the clicked location and the resultant location of the I-beam. 
the difference between the text sizes before and after repositioning the lens. Figure 6 demonstrates the problem: the cross hair in the left figure shows the location about to be clicked; the cross-hair in the right figure shows the unchanged location of the click; and the I-beam in the right-hand figure shows the resultant location of the insertion point. Consequently, having clicked at a particular point in the text, the user must search for the resultant (close-by) location of the I-beam. Users reported that although this was initially disturbing, they quickly became used to this small motion.

The second cause of text-motion was more disturbing for the users because it arose from the actions of their partners' and was therefore seen as 'unpredictable'. Whenever a colleague moved from a location above a user to a location below the user (or from below to above) their action would cause a small vertical offset in the user's view. This offset is due to the relocation of the colleague's lens. The effect is most noticeable when awareness of colleagues is set at high levels, and when the background text size is small. Equation 2 concisely describes the cause and magnitude of the effect. Methods of reducing this problem are discussed in Section 4.

\subsubsection{Location versus Semantic Distortion}

We observed several occurrences of subjects becoming disoriented in the document, typified by accompanying comments such as: "Now, where's the sub-section gone?" The problem was explicitly identified by one pair but, according to our observations, affected several pairs. It was caused by the absence of semantically oriented distortion in the fisheye lens.

DOME's fisheye lenses magnify text purely according to the vertical distance that the text lies from the centre of the lens. The distortion functions do not account for the semantic importance of the underlying textual units. Furnas used the term 'a priori importance' to capture this notion of semantic importance within information spaces (Furnas, 1986). In written articles there is a high level of a priori importance associated with the structural tags of the document such as section headings, titles, captions and so on. Users who are familiar with WYSIWIS word processors will expect to see these document elements displayed in a format that is similar to their final rendering — normally large and emboldened text.

In our observations, there were clear periods of conflict between the text that the subjects' expected to see magnified and the text that was magnified. The problem was particularly pronounced when the subjects had selected extremely small background text-sizes, and when section or subsection headings rolled out of the magnified region of the lens.

\section{Discussion and Further Work}

We are not overly discouraged by the results of our evaluation. We believe that the worst problem encountered by the subjects (the unwanted vertical text motion caused by the actions of partners) can be significantly reduced or eliminated by ensuring that the vertical location of the insertion point remains constant whenever lenses move. Small amendments to the vertical scroll location can be used to correct the $\delta_{y}$ offset (see Equation 2). Retaining the horizontal location of the insertion point is more problematical. One approach to correcting the $\delta_{x}$ offset would be to use horizontal scrolling. This is unlikely to be acceptable, however, because part of the text line 
would have to be scrolled out of the viewable area. Wide margins at low levels of magnification could overcome the need for scrolling, but they would be extremely wasteful of screen real-estate. Other potential solutions would be to dynamically modify the line-wrap of the text, but this is also unlikely to be popular because of the amount of dynamic text-modification that would be necessary.

We will not correct horizontal displacement ( $\delta_{x}$ offsets) in the next version of DOME. In our study, it was the vertical offsets caused by other users that most severely disrupted interaction. Subsequent evaluations will determine whether fixing the vertical offsets is sufficient for user acceptance.

The 'location versus semantic content' problem can be viewed as an issue of insufficient functionality in the current version of the system. DoME already has the ability to parse and amend the semantic units within $\mathrm{AT}_{\mathrm{E}} \mathrm{X}$ documents and $\mathrm{TCl} / \mathrm{Tk}$ programs, and consequently it should not be difficult to add magnification functions based on a priori importance: for instance, ensuring that section or procedure headings are always displayed larger than the surrounding text.

Having made these modifications to the system we will re-initiate evaluation in a similar style to that described in Section 3. Provided that the modifications result in a system that is acceptable to the users, we will undertake a longitudinal evaluation study to investigate how the awareness features are used by collaborators who are thoroughly familiar with the system.

\section{Related Work}

Research on distortion-oriented visualizations and on fisheye views stems from Furnas's (1986) seminal work which describes a mechanism for representing the user's "neighbourhood in great detail, yet only major landmarks further away". A recent review of distortion-oriented presentation techniques can be found in Leung $\&$ Apperley (1994). The work reported here is concerned with a new application area for distortion-oriented techniques: the provision of workspace awareness.

In one of the earliest papers on the importance of mutual awareness in synchronous groupware environments Stefik et al. (1987) reported on a variety of WYSIWIS mechanisms for providing focused interfaces for collaboration. The substantial usability problems of their Colab meeting environment were later reported in Tatar et al. (1991). The problems arose from users misunderstanding exactly what each other could see on their screens, and from mistaken deictic references.

Within editing environments Dourish \& Bellotti (1992) note the dual requirements of "understanding the activities of others" while maintaining a "context for your own activity". Towards this end, Gutwin et al. (1996) explored the development and usability of widgets specifically designed to provide an awareness of other users in the workspace. These widgets include 'shared telepointers' which show the precise location of each user within the workspace and 'gestalt-viewers' which provide a miniature overview of the entire workspace.

Our previous work (Greenberg et al., 1996a; Greenberg et al., 1996b; Greenberg et al., 1996c) demonstrated a variety of distortion-oriented mechanisms for workspace awareness in text-based and graphical workspaces. The systems described in these works were purely intended to demonstrate the types of awareness 
that distortion-oriented mechanisms could provide, and they were inadequate for evaluation.

May \& Barnard (1995) provide an interesting analysis of problems associated with "presentationally complex interfaces". Their work, which derives interface design guidance from an Interacting Cognitive Subsystems analysis of cinematography, would have predicted the problems that DOME users encountered with text movement.

\section{Conclusions}

Distortion-oriented visualization techniques appear to have the potential to satisfy collaborators' needs for workspace awareness in real-time groupware environments. They allow users to focus on their local area of work while simultaneously offering a viewport onto the work of colleagues. By tailoring the distortion provided by the visualization scheme, users can tailor their level of awareness of colleagues. The tailoring capability promises to bridge the gap between differing styles of collaborative work: from largely independent asynchronous collaboration, through semi-synchronous 'awareness', to tightly coupled and fully synchronous WYSIWIS collaboration.

Our distortion-oriented multi-user editor, Dome, has been designed and constructed to provide a fully-functional environment for experimenting with and evaluating distortion-oriented workspace awareness. The first evaluation of DomE, reported in this paper, illuminated several substantial usability problems with our implementation of awareness capabilities through 'fisheye' distortion. Although these problems overwhelmed the evaluation results, we are encouraged by evidence of successful uses of the awareness features. Our further work will ease the usability problems and continue evaluation.

\section{Acknowledgements and Availability}

Dome is written in Tcl/Tk (Ousterhout, 1993) and GroupKit (Roseman \& Greenberg, 1996). Its starting point was a fisheye text viewer developed by Saul Greenberg while on sabbatical at the University of Canterbury. Dome has been tested on Sun Sparc stations and on PCs running the Linux operating system. It is available on request from the second author.

\section{References}

Dourish, P. \& Bellotti, V. (1992), Awareness and Coordination in Shared Workspaces, in J. Turner \& R. Kraut (eds.), Proceedings of CSCW'92: Conference on Computer Supported Cooperative Work, ACM Press, pp.107-14.

Furnas, G. W. (1986), Generalized Fisheye Views, in M. Mantei \& P. Orbeton (eds.), Proceedings of CHI'86: Human Factors in Computing Systems, ACM Press, pp.16-23.

Greenberg, S., Gutwin, C. \& Cockburn, A. (1996a), “Applying Distortion-oriented Displays to Groupware", Video Programme of the ACM Conference on Computer Supported Cooperative Work. 
Greenberg, S., Gutwin, C. \& Cockburn, A. (1996b), Awareness Through Fisheye Views in Relaxed-WYSIWIS Groupware, in Proceedings of 1996 Graphics Interface Conference., Morgan-Kaufmann, pp.28-38.

Greenberg, S., Gutwin, C. \& Cockburn, A. (1996c), Using Distortion-oriented Displays to Support Workspace Awareness, in A. Sasse, R. J. Cunningham \& R. Winder (eds.), People and Computers XI (Proceedings of HCI'96), Springer-Verlag, pp.299-314.

Greenberg, S., Gutwin, C. \& Roseman, M. (1996d), Semantic Telepointers for Groupware, in J. Grundy \& M. Apperley (eds.), Proceedings of OzCHI'96 The Sixth Australian Conference on Computer-Human Interaction, IEEE Computer Society Press, pp.54-61.

Gutwin, C., Roseman, M. \& Greenberg, S. (1996), A Usability Study of Awareness Widgets in a Shared Workspace Groupware System, in M. S. Ackerman (ed.), Proceedings of CSCW'96: Conference on Computer Supported Cooperative Work, ACM Press, pp.25867.

Leung, Y. K. \& Apperley, M. (1994), “A Review and Taxonomy of Distortion-Oriented Presentation Techniques", ACM Transactions on Computer-Human Interaction 1(2), 126-60.

May, J. \& Barnard, P. (1995), Cinematography and Interface Design, in K. Nordby, P. H. Helmersen, D. J. Gilmore \& S. A. Arnessen (eds.), Human-Computer Interaction - INTERACT'95: Proceedings of the Fifth IFIP Conference on Human-Computer Interaction, Chapman \& Hall, pp.26-31.

Ousterhout, J. K. (1993), An Introduction to Tcl and Tk, Addison-Wesley.

Roseman, M. \& Greenberg, S. (1996), "Building Real Time Groupware with GroupKit, A Groupware Toolkit”, ACM Transactions on Computer-Human Interaction 3(1), 66-106.

Stefik, M., Bobrow, D. G., Foster, G., Lanning, S. \& Tatar, D. (1987), "WYSIWIS Revised: Early Experiences with Multiuser Interfaces", ACM Transactions on Office Information Systems 5(2), 147-67.

Tatar, D. G., Foster, G. \& Bobrow, D. G. (1991), "Design for Conversation: Lessons from Cognoter”, International Journal of Man-Machine Studies 34(2), 185-209. 


\section{Author Index}

Cockburn, Andy, 1

Weir, Philip, 1 

Keyword Index

collaborative workspace awareness, 1

groupware, 1

distortion-oriented visualization, 1 